\title{
Novel Six-Quark Hidden-Color Dibaryon States in QCD
}

\author{
M. Bashkanov ${ }^{\mathrm{a}, *}$, Stanley J. Brodsky ${ }^{\mathrm{b}}, \mathrm{H}$. Clement ${ }^{\mathrm{a}}$ \\ ${ }^{a}$ Physikalisches Institut, Eberhard-Karls-Universität Tübingen, Auf der Morgenstelle 14, 72076 Tübingen, Germany \\ ${ }^{b}$ SLAC National Accelerator Laboratory Stanford University
}

\begin{abstract}
The recent observation of a hadronic resonance $d^{*}$ in the proton-neutron system with isospin $I=0$ and spin-parity $J^{P}=3^{+}$raises the - possibility of producing other novel six-quark dibaryon configurations allowed by QCD. A dramatic example of an exotic six-quark color-singlet system is the charge $Q=+4$, isospin $I=3, I^{z}=+3 \mid$ ииииии $>$ state which couples strongly to $\Delta^{++}+\Delta^{++}$. The width and decay properties of such six-quark resonances could be regarded as manifestations of "hidden-color" six-quark configurations, a first-principle prediction of QCD - SU(3)-color gauge theory for the deuteron distribution amplitude. Other implications and possible future experiments are discussed.
\end{abstract}

'Keywords: exotic hadrons; hidden color, hexaquark states

\section{Introduction}

Because of color confinement, one expects that virtually any color-singlet hadronic configuration of quarks and gluons can form either bound states or resonances. In addition to the familiar $q \bar{q}$ mesons, $q q q$ baryons, the $g g$ and $g g g$ glueball 'states [1], as well as nuclei, color confinement can lead to $q \bar{q} q \bar{q}$ "tetraquark" systems [2] such as the charged $Z_{c}(c \bar{c} u \bar{d})$ [3, 4] 'and possibly $q q q q \bar{q}$ "pentaquark" states [5]. Mesonic nuclei $[6,7,8,9,10,11,12]$ and nuclear-bound quarkonium [13, 14, 15] are also possible. Resonances in the $\bar{q} \bar{q} q q q$ channel just below the $B \bar{B}$ threshold could explain the anomalously large rates [16] seen in $e^{+} e^{-} \rightarrow p \bar{p}, n \bar{n}, \bar{\Lambda} \Lambda$ at threshold. The anomalously large transverse spin-spin correlation $A_{N N}$ observed in large-angle proton-proton elastic scattering near the strangeness and charm thresholds [17] could be explained by the effects of $\mid$ uuduud $Q \bar{Q}>$ baryon number $B=2$ resonances in the $J=L=1 p p s$-channel [18, 19].

Understanding the mechanisms underlying confinement in QCD is among the most fundamental questions in hadron physics. In the case of heavy quarks, the potential evidently can -be identified with gluon exchange, in analogy with the Coulomb forces which bind atoms. The potential underlying light-quark interactions is however much more complex - such as fluxtube exchange [20] and other string-like forces [21] built from multi-gluon exchange. It has recently been shown that the effective confining $q \bar{q}$ potential in the frame-independent QCD lightfront (LF) Hamiltonian has a unique form [22] if one maintains conformal symmetry of the QCD action, The resulting meson eigensolutions of the resulting light-front Schrodinger equation include a zero-mass pion in the chiral $m_{q} \rightarrow 0$ limit, and linear Regge trajectories $M^{2}(n, L) \propto n+L$ with the same slope

\footnotetext{
${ }^{*}$ Corresponding author

Email address: bashkano@pit.physik.uni-tuebingen.de (M. Bashkanov)
}

in the radial quantum number $n$ and orbital angular momentum $L$. In the case of light baryons, the confining potential could mimic the $q \bar{q}$ form as a two-body quark-diquark interaction in a light-front Dirac equation [24, 23], or take the form of a three-body force such as a $Y$ junction [25] between the valence quarks. The LF Dirac equation based on quark-diquark interactions with the same potential as $q \bar{q}$ accounts well for the measured light baryon spectrum [23]

The possible mechanisms underlying confinement multiply as the number of quarks and gluon constituents in a hadronic system increase. A key question is whether such states bound by fundamental QCD interactions or do the constituents always cluster as color-singlet subsystems? In the case of nuclei, the quark constituents evidently cluster as color-singlet nucleons bound by virtual meson exchange, the analog of covalent binding in molecular physics due to quark interchange or exchange. When there are no covalence quarks in common, QCD also predicts attractive multigluonic van der Waals forces which are dual to glueball exchange. The attractive QCD van der Waals potential leads to the prediction of bound states of heavy quarkonium to heavy nuclei [13, 14, 26]. However, there are also rare configurations in which other multiquark color configurations ("hidden color" [27]) can enter.

There are several possible interpretations [28] for the dominant internal structure of the positively charged $Z_{c}^{+}(4025)$, which can be identified as a $\mid c \bar{c} u d>$ color-singlet tetraquark bound state. The $Z_{c}$ could be considered an example of a bound state of $c \bar{c}$ quarkonium with a light $u \bar{d}$ meson bound by gluon exchange, corresponding to "disconnected contributions" in lattice gauge theory simulations [29]); or a $D^{*} \bar{D}^{*}$ hadronic molecule [30, 2, 31] such as $D^{*}(c \bar{u}) \bar{D}^{*}(\bar{c} d)$ clusters bound by meson exchange. Other color-confining interactions between higher-color multiquark representations may also dominate [32, 33].

The possibility of exotic six-quark qqqqqq dibaryonic "hex- 
aquark" states was first proposed by F. J. Dyson and N. Xuong [34] in 1964, just a half a year after Gell-Mann's publication of the quark model [35]. However, this topic received intensive attention only after Jaffe's proposal[36] of the socalled "H dibaryon", a $\mid u u d d s s>$ state corresponding asymptotically to a bound $\Lambda \Lambda$ system. This hypothesis initiated a worldwide activity of theoretical predictions for dibaryon states with and without strangeness - as well as numerous experimental searches. Despite numerous claims, no established dibaryon candidate has emerged. For a recent report concerning the experimental $\mathrm{H}$ dibaryon search see e.g. Ref. [37]. However, there has been renewed interest in such states, in part because lattice QCD calculations are now becoming available [38, 39, 40, 41, 42, 43, 44].

As we shall show in this paper, the discovery of six-quark states would provide a novel extension of the domain of hadronic states in QCD, and the experimental verification of such dibaryon states may well be possible in the near future.

The most familiar six-quark state is the isospin-zero $\mid u u d d d u>$ deuteron; in fact, the wavefunction of the deuteron has novel properties in QCD. Five distinct color-singlet configurations of six color triplets $3_{C}$ can form a color singlet in $S U(3)$ color, only one of which corresponds to the usual $p n$ configuration. When one probes the light-front wavefunctions of the deuteron where all of the six quarks have small relative separation, as in the deuteron form factor at high moment transfer or in photodisintegration $\gamma d \rightarrow n p$ at high transverse momentum, the five "hidden-color configurations" of the deuteron mix due to gluon exchange and become equal in magnitude at asymptotic $Q^{2} \rightarrow \infty$ [27]. For example, the observed $Q^{10} F_{d}\left(Q^{2}\right)$ scaling [45] of the deuteron $\sqrt{A}\left(Q^{2}\right)$ form factor at high $Q^{2}$ [46] is dominated by hidden-color configurations. This result can be derived by applying ERBL evolution [47, 48] to the five-component deuteron distribution amplitude $\phi_{d}\left(x_{i}, Q\right)$. The color-singlet states of the deuteron wavefunction also couple to a virtual $\Delta^{+} \Delta^{0}$ state [49].

The most dramatic example of an exotic six-quark colorsinglet system is the charge $Q=+4$, isospin $I=3, I^{z}=$ $+3 \mid$ ииииии > state, as originally proposed by Dyson and Xuong [34]. The Fermi-Dirac statistics of the color-triplet $u$ quark only allows one color-singlet six-quark configuration with zero orbital angular momentum: $\mid u_{R}^{\uparrow} u_{Y}^{\uparrow} u_{B}^{\uparrow} u_{R}^{\downarrow} u_{Y}^{\downarrow} u_{B}^{\downarrow}>$. The set of seven $I^{z}=3,2,1,0,-1,-2,-3$ states ranging from $Q=+4$ to $Q=-2$ are then obtained by applying the isospinlowering operator. As a first approximation, one can estimate their masses $\simeq 2.4 \mathrm{GeV}$ by considering these states as effective $\Delta \Delta$ bound states; e.g. the $\mid и и и и и и>$ state can be considered as a bound-state of two $I^{z}=3 / 2 \Delta^{++}$isobars - for model predictions of its mass see, e.g. Refs. [34, 50, 51, 52, 53, 54, 55]. We do not expect major Coulombic corrections to its dibaryon properties from the high $Q=+4$ charge since one does not observe significant charge-related effects in $n n-p p$ or in $\Delta^{++}-\Delta^{0}$ systems.

In this paper we will review the present evidence for dibaryon states and discuss future strategies for detecting such six-quark states. A typical example is the study of $p p \rightarrow \pi^{-} \pi^{-} X$, where the recoil system $X$ could display a charge $Q=+4$ resonance peak in the $X$ missing mass. One can also look for $\Delta^{++} \Delta^{++}$ resonance decay as an enhancement in the rate of the exclusive channel measurement $p p \rightarrow \pi^{-} \pi^{-} \Delta^{++} \Delta^{++}$. The enhancement could appear below the nominal two-isobar mass, indicating a possible $\Delta \Delta$ bound state phenomenon.

\section{Recent Experimental Evidence for a $\Delta \Delta$ Resonance}

A pronounced resonance structure has recently been observed in $p n$ collisions leading to two-pion production in the reactions $p n \rightarrow d \pi^{0} \pi^{0}$ [57, 58], $p n \rightarrow d \pi^{+} \pi^{-}$[59], $p n \rightarrow$ $p p \pi^{-} \pi^{0}$ [60] and possibly also in pn elastic scattering, in particular in the total cross section and in the analyzing power [61]. For the not yet measured reactions $p n \rightarrow p n \pi^{0} \pi^{0}$ and $p n \rightarrow p n \pi^{+} \pi^{-}$exist predictions for the size of the expected resonance effect [62, 63].

The measured parameters for this resonance structure, called henceforth $d^{*}$, are $I\left(J^{P}\right)=0\left(3^{+}\right)$with mass $M=2.37 \mathrm{GeV}$ and width $\Gamma=70 \mathrm{MeV}$ [58, 59, 60]. Dalitz plots indicate that $d^{*}$ dominantly decays via an intermediate $\Delta-\Delta$ system. However, the mass of this resonance is about $90 \mathrm{MeV}$ below the nominal mass $2 m_{\Delta}$ of a $\Delta \Delta$ system, and its width is about three times smaller than that of a $\Delta \Delta$ system formed by conventional $t$-channel meson exchange or quark interchange arising within the $N N$ collision processes. The interchange of quarks of the same flavor [64] has been shown to dominate hadron-hadron elastic scattering amplitudes in the hard-scattering fixed $\theta_{C M}$ scattering domain [65].

We conclude from such observations that $d^{*}$ must be of an unconventional origin, possibly indicating a genuine six-quark nature. With the predominant decay of $d^{*}$ being $d^{*} \rightarrow \Delta \Delta$ $\left(B R\left(d^{*} \rightarrow \Delta \Delta\right) / B R\left(d^{*} \rightarrow p n\right)=9: 1\right)$, one could naively expect $d^{*}$ to be a so-called a "deltaron" denoting a deuteron-like bound state of two $\Delta \mathrm{s}$. However, the narrow width of $d^{*}$ contradicts this simple assumption. A deltaron would need to have 90 $\mathrm{MeV}$ binding energy, i.e. $45 \mathrm{MeV}$ per $\Delta$, which would lead to a reduction of width from $\Gamma_{\Delta \Delta}=230 \mathrm{MeV}$ to $\Gamma_{\Delta \Delta}=160 \mathrm{MeV}$, using the known momentum dependence of the width of the $\Delta$ resonance. This is more than twice what is observed.

On the other hand, if $d^{*}$ is a genuine six-quark dibaryon state, we need to understand its large coupling $d^{*} \rightarrow \Delta \Delta$. This can be explained if one assumes the $d^{*}$ is dominated by a "hiddencolor" six-quark state. Hidden-color six-quark states are a rigorous first-principle prediction of SU(3) color gauge theory. Six quark color-triplets $3_{C}$ combine to five different color-singlets in QCD, - and as shown in Ref. [27], will significantly decay to $\Delta \Delta$.

According to M. Harvey [66] there are only two possible quark structures for an $I\left(J^{P}\right)=0\left(3^{+}\right)$resonance in the twobaryon system:

$$
\begin{aligned}
& \left|\Psi_{d^{*}}\right\rangle=\sqrt{\frac{1}{5}}|\Delta \Delta\rangle+\sqrt{\frac{4}{5}}|6 Q\rangle \text { and } \\
& \left|\Psi_{d^{*}}\right\rangle=\sqrt{\frac{4}{5}}|\Delta \Delta\rangle-\sqrt{\frac{1}{5}}|6 Q\rangle .
\end{aligned}
$$

Here $\Delta \Delta$ means the asymptotic $\Delta \Delta$ configuration and $6 Q$ is the genuine "hidden color" six-quark configuration. The first 
solution denotes a $S^{6}$ quark structure (all six quarks in the $\mathrm{S}$ shell), the second one a $S^{4} P^{2}$ configuration (4 quarks in the $\mathrm{S}$ shell and 2 quarks in the P-shell). The quark structure with the large $\Delta \Delta$ coupling would correspond to a deltaron and can be excluded. Thus it is natural to assign the observed $d^{*}$ resonance to the $S^{6}$ six-quark predominantly "hidden color" state, thus providing an explanation for its narrow decay width.

The above ansatz for the $d^{*}$ wavefunction and the decay of $d^{*}$ via the ${ }^{7} S_{3}$ configuration of the intermediate $\Delta^{+} \Delta^{0}$ system imply that the resulting nucleon and pion pairs are either both in $\mathrm{I}=0$ or both in $\mathrm{I}=1$ isospin states. The first possibility is realized in the experimentally studied channels $d \pi^{0} \pi^{0}[58]$ and $d \pi^{+} \pi^{-}$[59], and the second possibility can be studied in the $p p \pi^{0} \pi^{-}$channel, where a signal for the $d^{*}$ has been observed, too [60]. In the $\mathrm{I}=1$ case the pion pair must be in relative $p$ wave in order to comply with Bose statistics; in addition, the nucleon pair must be in a relative $p$-wave or higher in order to obtain the required spin and parity of the $d^{*}$. Higher orbital angular momenta cannot be excluded at present, requiring a more complicated wavefunction than given above.

Due to its quantum numbers, the $d^{*}$ state must be fully symmetric in spin, color, and angular momentum as well as fully antisymmetric in isospin. Due to this particular feature, Ref. [51] claims that any model based on confinement and effective onegluon exchange leads to the prediction of the existence of a non-strange dibaryon with $I\left(J^{P}\right)=0\left(3^{+}\right)$, the "inevitable nonstrange dibaryon". In fact, many groups [34, 50, 51, 52, 53, 54] predicted such a state at similar mass. Very recently Gal and Garcilazo succeeded to predict such a deeply bound $\Delta \Delta$ state at the experimentally observed mass in a fully relativistic threebody calculation based on hadron dynamics 67.

It is remarkable that the first such calculation published by Dyson and Xuong [34 appears now to be quite precise in the prediction of the $d^{*}$ mass. In the nomenclature of Ref. [34], the $d^{*}$ has the notation $D_{03}$, where the indices (03) denote the isospin $I=0$ and spin $J=3$ of the dibaryon. To predict the mass of $D_{03}$ Dyson and Xuong identified the $D_{01}$ state with the ${ }^{3} S_{1}$ deuteron ground-state and the $D_{10}$ with the ${ }^{1} S_{0}$ virtual state (unbound by $66 \mathrm{keV}$ only [68]), which is known to contribute to the nucleon-nucleon final-state interaction. These two states are also currently being used to check the reliability of lattice calculations for the H-dibaryon [40, 42, 44, 69, 70].

Most quark models predict [34, 50, 51, 52, 53, 54] that in addition to $d^{*}$ one should have also a state with mirrored quantum numbers for spin and isospin, i.e. $I\left(J^{P}\right)=3\left(0^{+}\right)$at a similar mass. Such a state, which in the notation of Ref. [34] is $D_{30}$, would be symmetric in isospin, color, angular momentum and antisymmetric in spin. Due to its isospin $I=3$, it cannot decay into $N N$ or $N N \pi$, but only into the $N N \pi \pi$ channel. Thus if such a state has a mass close to that of $d^{*}$, its width must be even smaller than that of $d^{*}$.

According to Ref. [34], both $d^{*}$ and $D_{30}$ belong to multiplets of dibaryons, the first one is assigned to an antidecuplet and the second one to a 28-plet. Thus, given the existence of the $d^{*}$, one should expect a number of strange dibaryons. The three corners of the possible 28-plet look truly exotic: 6u quarks, 6d quarks, 6 s quarks. In each of these cases the quarks occupy all possible states. The $6 \mathrm{~s}$ quark state can be considered as a strange droplet and could play an important role in astrophysics regarding the nuclear equation of matter in the core of neutron stars. Recent calculations on $\Omega \Omega$ (6s quark state) [71, 72, 73] display a range of results - from $100 \mathrm{MeV}$ binding to an unbound state.

\section{Experimental Strategies}

The existence of novel dibaryon states still awaits definitive experimental confirmation or exclusion. Thus we will discuss in the following a number of possible experiments and strategies for producing the charge- $1 d^{*}$ and charge- $4 D_{30}$, such as electro- and photo-production of $d^{*}$ on a deuteron: $\gamma d \rightarrow d^{*} \rightarrow$ $d \pi^{0} \pi^{0}$. A suitable place to perform such an experiment appears to be MAMI at Mainz due to its high beam intensity and good neutral particles detection capabilities of the Crystal Ball experiment. Such a reaction should preferably go via photon coupling to the deuteron's six-quark component and will allow to fix the transition from the six-quark component in the deuteron to the one of $d^{*}$. The reaction $\gamma d \rightarrow d^{*} \rightarrow d \pi^{+} \pi^{-}$is less favorable due much higher background rates [74]. For a first calculation of the $d^{*}$ electroproduction see Ref. [75].

Due to the expected small cross sections, such experiments require sufficient beam intensity as well as large detector acceptance for photons and efficient particle identification in order to discriminate deuterons from protons. Several detector setups fulfill these conditions: the Crystal Ball at MAMI/Mainz [76], the Crystal Barrel [77] and BGO-OD at Elsa/Bonn. The use of a polarized photon beam and a polarized deuteron target at the Crystal Ball and Crystal Barrel experiments can provide further suppression of the conventional background. In addition, the Crystal Ball, in combination with a nucleon recoil polarimeter [78], can investigate the $\gamma d \rightarrow d^{*} \rightarrow \vec{p} n$ reaction where a sign of the $d^{*}$ resonance was observed in the seventies [50, 79].

With the knowledge of the $d d^{*} \gamma$ coupling one can estimate possible cross sections for the production of other antidecuplet members in reactions like $\gamma d \rightarrow d_{s}^{*}+K^{+} \rightarrow \Delta \Sigma^{*}+K^{+}$. Such reactions could be measured at JLab. Another possibility to produce the strange partners of $d^{*}$ would be the study of kaoninduced reactions of the kind $K^{-} d \rightarrow d_{s}^{*} \rightarrow \Delta \Sigma^{*}$ as could be conducted at JPARC.

Accessing the members of the 28-plet appears to be much more complex. Most prominent here is $D_{30}$ with charge $Q=+4$ (six u-quarks). The dedicated decay channel of such a state is $D_{30} \rightarrow p p \pi^{+} \pi^{+}$which can be triggered with high selectivity. However, the production of such a state is challenging. One may be able to produce it in $p p$ collisions; however, in order to reach the $I=3$ state, one needs to produce in addition two associated negative pions $p p \rightarrow D_{30} \pi^{-} \pi^{-} \rightarrow\left(p p \pi^{+} \pi^{+}\right) \pi^{-} \pi^{-}$. To perform such a reaction in the energy region of interest, one needs a rather high beam energy of $T_{p}=1.7-2 \mathrm{GeV}$ which is available at COSY and JPARC. However, the $p p \pi^{+} \pi^{+} \pi^{-} \pi^{-}$ channel will be highly contaminated by conventional $N^{*}$ and $\Delta$ excitations.

Another important way to identify the $D_{30}$ is its production in nuclei, e.g. on carbon by the reaction $\gamma C^{12} \rightarrow p p \pi^{+} \pi^{+} X$ below the $4 \pi$ threshold at JLab, or similarly using proton or 
pion beams in reactions such as $p^{12} \mathrm{C} \rightarrow p p \pi^{+} \pi^{+} X$ and $\pi^{+12} \mathrm{C} \rightarrow$ $p p \pi^{+} \pi^{+} X$. In all such reactions the conventional background due to associated meson production production needs to be effectively suppressed. Magnetic separation of positively and negatively charged pions is a prerequisite for measuring such reactions due to the much higher $\pi^{+} \pi^{-}$rate. The CLAS detector [80] at JLab with toroidal magnetic field, large acceptance and high momentum resolution would be a very suitable place to perform such experiments.

The detection of the $Q=+4 D_{30}$ resonance would help to constrain the properties of the "strange droplets", the $\Omega \Omega$ states, and thus simplify its search in heavy-ion collisions.

Another place to look for both the $D_{03}\left(d^{*}\right)$ and $D_{30}$ resonances is to search in quarkonium decays. The high mass of dibaryonic resonances excludes charmonium decays; however, bottomium decays measured at B-factories appear to be promising. The observation of the $d^{*}$ looks particularly straightforward: due to its isospin $I=0$ one does not necessarily need to search for $\Upsilon \rightarrow \bar{d}^{*} d^{*}$; the search for $\Upsilon \rightarrow \bar{d} d^{*}$ would be sufficient. The branching ratio of $B R(\Upsilon \rightarrow \bar{d}+X)=2.86 \times 10^{-5}[81$ ] appears to be large enough to search for the reaction $\Upsilon \rightarrow \bar{d} d^{*}$ or $\bar{d}^{*} d \rightarrow d \bar{d}(\pi \pi)_{I=0}$. This simple possibility is forbidden for the $D_{30}$ because of its isospin. One could produce the $D_{30}$ paired with $\bar{D}_{30}$ having in minimal configuration $\Upsilon \rightarrow \bar{D}_{30} D_{30} \rightarrow$ $\left(\bar{p} \bar{p} \pi^{-} \pi^{-}\right)\left(p p \pi^{+} \pi^{+}\right)$. Unfortunately, this channel will contain large contamination from the production of conventional $N^{*}$ and $\Delta$ resonances and their antimatter analogs. However, one can extract not only the mass and width of resonances in this way, but also its time-like form-factor. The extraction of the space-like form-factor for such a state appears to be impossible at the present level of experimental capabilities, so distinguishing between molecular-type and genuine dibaryon will be challenging.

To our knowledge dibaryon channels have not yet been looked for at $e^{+} e^{-}$colliders; however, the statistics of data already collected at BaBar and Belle should be large enough to search for such resonances. Recent publications on the search for H-dibaryon production in the $\Upsilon$ decays by Belle [37] may be a good starting point for the search of other dibaryon candidates, including the ones discussed here.

Another important experimental option is the photoproduction or electroproduction of a dibaryon state on a nucleon target in combination with associated anti-nucleon production, such as $\gamma p \rightarrow \bar{p} \pi^{-} \pi^{-} D_{30} \rightarrow\left(\bar{p} \pi^{-} \pi^{-}\right)\left(p p \pi^{+} \pi^{+}\right)$, a reaction which could be investigated at the upcoming $12 \mathrm{GeV}$ electron facility at JLab. The advantage of such reactions is the particularly simple triggering conditions - the essential signal for the dibaryon is provided by the antiproton trigger. Of course, as in the other cases discussed above, one may encounter a high level of conventional backgrounds.

The triggering on antiparticles promises to be even better suited for strange dibaryons. In case of strangeness $S=-1$, the tagging on the $\overline{\Sigma^{*}}$ allows one to separate antidecuplet from 27 plet states. Only a 27 -plet $J=0$ state can be produced in combination with $\overline{\Sigma^{*-}}$ in the reaction $\gamma p \rightarrow \overline{\Sigma^{*-}}+\left(\Sigma^{*+} \Delta^{+}\right)_{27}$, whereas with $\overline{\Sigma^{*+}}$ both the 27 -plet $J=0$ and antidecuplet $J=3$ states are possible in the process $\gamma p \rightarrow \overline{\Sigma^{*+}}+\left(\Sigma^{*-} \Delta^{+}\right)_{27,10}$. Similarly, one can search for double and triple-strange dibaryons in the reactions $\gamma p \rightarrow \overline{\Xi^{*+}}+\left(\Xi^{*-} \Delta^{+}\right)_{27,10}$ and $\gamma p \rightarrow \bar{\Omega}+\left(\Omega \Delta^{+}\right)_{27, \overline{10}}$. in addition, tagging on antiparticles can effectively suppress conventional backgrounds.

\section{Summary}

The recent observation of a narrow hadronic proton-neutron resonance $d^{*}$ with $I\left(J^{P}\right)=0\left(3^{+}\right)$and mass $M=2.37 \mathrm{GeV}$ raises the possibility of producing other novel color-singlet six-quark dibaryon configurations allowed by QCD. A dramatic example would be the discovery of an exotic six-quark $\mid$ ииииии > colorsinglet system with charge $Q=+4$, isospin $I=3$, and $I^{z}=+3$, a state which couples strongly to $\Delta^{++}+\Delta^{++}$. The width and decay properties of such six-quark resonances could be regarded as a manifestation of either a "hidden-color" six-quark configuration, versus a more conventional interpretation as a $\Delta-\Delta$ (deltaron) resonance. We have discussed a number of possible experiments where such a state could be observed.

\section{Acknowledgments}

We are grateful to David Bugg, William Detmold, Avraham Gal, Johann Haidenbauer, Christoph Hanhart, Marek Karliner, Eulogio Oset and Colin Wilkin for helpful discussions. This work was supported by the Department of Energy contract DE-AC02-76SF00515, the BMBF (06TU9193) and the Forschungszentrzum Jülich (COSY-FFE). SLAC-PUB-15720.

\section{References}

[1] W. Ochs J. Phys. G 40 043001, 2013.

[2] M. Karliner and S. Nussinov, JHEP 1307, 153 (2013) arXiv:1304.0345 [hep-ph]].

[3] M. Ablikim et al., Phys. Rev. Lett. 110, 252001 (2013).

[4] Z.Q. Liu et al., Phys. Rev. Lett. 110, 252002 (2013).

[5] The nomenclature $q \bar{q}$ etc. refers to the lowest particle number Fock state of the hadronic eigensolution of the QCD light-front Hamiltonian.

[6] P. Adlarson et al., Phys. Rev. C 87, 035204 (2013)

[7] Akira Yokota, Emiko Hiyama, Makoto Oka arXiv:1308.6102 [nucl-th]

[8] E. Oset et al., Nucl.Phys. A881, 127, (2012)

[9] E. Friedman, A. Gal, J. MareÅą, Phys. Lett. B 725, 334, (2013)

[10] H. Nagahiro et al., Phys.Rev. C87, 045201 (2013)

[11] M. Bayar et al., Phys.Rev. C86, 044004 (2012)

[12] M. Kaskulov et al., Phys.Rev. C75 064616 (2007)

[13] S. J. Brodsky, I. A. Schmidt and G. F. de Teramond, Phys. Rev. Lett. 64, 1011 (1990).

[14] M. E. Luke, A. V. Manohar and M. J. Savage, Phys. Lett. B 288, 355 (1992) |hep-ph/9204219|.

[15] V.B. Belyaev, N.V. Shevchenko, A.I. Fix, W. Sandhas Nucl.Phys. A780 (2006) 100-111

[16] R. Baldini, S. Pacetti, A. Zallo and A. Zichichi, Eur. Phys. J. A 39, 315 (2009) [arXiv:0711.1725 [hep-ph]].

[17] G. R. Court, D. G. Crabb, I. Gialas, F. Z. Khiari, A. D. Krisch, A. M. T. Lin, R. S. Raymond and R. R. Raylman et al., Phys. Rev. Lett. 57, 507 (1986).

[18] S. J. Brodsky and G. F. de Teramond, Phys. Rev. Lett. 60, 1924 (1988).

[19] S. Brodsky, G. de Teramond and M. Karliner, Ann. Rev. Nucl. Part. Sci. 62, 1 (2012) arXiv:1302.5684 [hep-ph]].

[20] N. Isgur and J. E. Paton, Phys. Rev. D 31, 2910 (1985).

[21] Y. Makeenko, Phys. Lett. B 699, 199 (2011) [arXiv:1103.2269 [hep-th]].

[22] S. J. Brodsky, G. F. de Teramond and H. G. Dosch, arXiv:1302.4105 [hepth]. 
[23] S. J. Brodsky and G. F. de Teramond, PoS ConfinementX 128 (2012) arXiv:1301.2733 [hep-ph]].

[24] G. F. de Teramond and S. J. Brodsky, AIP Conf. Proc. 1432, 168 (2012) arXiv:1108.0965 [hep-ph]].

[25] T. T. Takahashi, H. Matsufuru, Y. Nemoto and H. Suganuma, Phys. Rev. Lett. 86, 18 (2001) |hep-lat/0006005|.

[26] K. YI, Int. J. Mod. Phys. A 28, 1330020 (2013) arXiv:1308.0772 [hepex]].

[27] S. J. Brodsky, C. R. Ji and G. P. Lepage, Phys. Rev. Lett. 51, 83 (1983); S. J. Brodsky and C. -R. Ji, Phys. Rev. D 33, 1406 (1986); Phys. Rev. D 34, 1460 (1986).

[28] N. Mahajan, arXiv:1304.1301 [hep-ph].

[29] F. -K. Guo, L. Liu, U. -G. Meis̈ner and P. Wang, arXiv:1308.2545 [heplat].

[30] M. B. Voloshin and L. B. Okun, JETP Lett. 23, 333 (1976) [Pisma Zh. Eksp. Teor. Fiz. 23, 369 (1976)].

[31] C. -F. Qiao and L. Tang, arXiv:1308.3439 [hep-ph].

[32] S. Weinberg, Phys. Rev. Lett. 110, 261601 (2013) arXiv:1303.0342 [hep$\mathrm{ph}]$.

[33] R. F. Lebed, arXiv:1308.2657[hep-ph].

[34] F. J. Dyson and N.-H Xuong, Phys. Rev. Lett. 13, 815 (1964).

[35] M. Gell-Mann, Phys. Lett. 8, 214 (1964).

[36] R. L. Jaffe, Phys. Rev. Lett. 38, 195 and 617(E) (1977).

[37] B. H. Kim et al. (Belle Collaboration) Phys. Rev. Lett 110222002 (2013).

[38] S. R. Beane et al., Phys. Rev. Lett.106 (2011) 162001.

[39] T. Inoue et al., Phys. Rev. Lett.106 (2011) 162002.

[40] S. R. Beane et al., Phys.Rev. D85, 054511 (2012)

[41] S. R. Beane et al., Mod.Phys.Lett. A26, 2587 (2011)

[42] S. R. Beane et al., Phys.Rev. D87, 034506 (2013)

[43] T. Inoue arXiv:hep-lat 1212.4230

[44] T. Inoue, S. Aoki et al., Nucl. Phys. A 881 28-43 (2012)

[45] S. J. Brodsky and B. T. Chertok, Phys. Rev. Lett. 37, 269 (1976).

[46] S. Rock, R. G. Arnold, P. E. Bosted, B. T. Chertok, B. A. Mecking, I. A. Schmidt, Z. M. Szalata and R. York et al., Phys. Rev. D 46, 24 (1992).

[47] S. J. Brodsky, G. P. Lepage, Phys. Lett. B 87, 359 (1979); Phys. Rev. D 22 (1980) 2157.

[48] A.V. Efremov, A.V. Radyushkin, Theor. Math. Phys. 42, 97 (1980); Phys. Lett. B 94 245, (1980).

[49] T. Frick, S. Kaiser, H. Muther and A. Polls, Phys. Rev. C 64, 014309 (2001) nucl-th/0101028.

[50] T. Kamae and T. Fujita, Phys. Rev. Lett. 38, 471 (1977).

[51] T. Goldman, K. Maltman, G.J. Stephenson, K.E. Schmidt, Fan Wang, Phys. Rev. C 39, 1889 (1989); J. L. Ping, H. X. Huang, H. R. Pang, F. Wang, C. W. Wong, Phys. Rev. C 79, 024001 (2009)

[52] P.J.G. Mulders and A. W. Thomas, J. Phys. G 9, 1159 (1983)

[53] A. Th. M. Aerts, P.J.G. Mulders, J.J. deSwart, Phys. Rev. D 17, 260 (1978)

[54] K. Maltman, Nucl. Phys. A 438, 669 (1985)

[55] R. D. Mota, A. Valcarce, F. Fernandez, D. R. Entem and H. Garcilazo, Phys. Rev. C 65, 034006 (2002)

[56] Y. Frishman and M. Karliner, arXiv:1305.6457[hep-ph].

[57] M. Bashkanov et al., Phys. Rev. Lett. 102, 052301 (2009).

[58] P. Adlarson et al., Phys. Rev. Lett. 106, 242302 (2011).

[59] P. Adlarson et al., Phys. Lett. B 721, 229 (2013)

[60] P. Adlarson et al., arXiv:1306.5130

[61] M. Bashkanov et al., presented at Baryon 2013, Glasgow

[62] C. Fäldt, C. Wilkin, Phys. Lett. B 701, 619 (2011)

[63] M. Albaladejo, E. Oset, Phys. Rev. C 88, 014006 (2013)

[64] J. F. Gunion, S. J. Brodsky and R. Blankenbecler, Phys. Rev. D 8, 287 (1973).

[65] C. White, R. Appel, D. S. Barton, G. Bunce, A. S. Carroll, H. Courant, G. Fang and S. Gushue et al., Phys. Rev. D 49, 58 (1994).

[66] M. Harvey Nucl. Phys. A 352, 301 (1979)

[67] A. Gal and H. Garcilazo, Phys. Rev. Lett., in press; arXiv:1308.2112 [nucl-th].

[68] V. V. Flambaum, R.B. Wiringa Phys. Rev. C 76, 054002 (2007)

[69] T. Yamazaki, Y. Kuramashi, A. Ukawa, Phys. Rev. D 84054506 (2011)

[70] T. Yamazaki, K. Ishikawa, Y. Kuramashi, A. Ukawa, Phys. Rev. D 86 074514 (2012)

[71] Z. Y. Zhang, Y.W. Yu, C. R. Ching, T. H. Ho, and Z. D. Lu, Phys. Rev. C 61, 065204 (2000)
[72] F. Wang, J. L. Ping, G. H. Teng and J. T. Goldman, Phys. Rev. C 51, 3411 (1995)

[73] M. I. Buchoff, T.C. Luu, J. Wasem, arXiv:1201.3596 1

[74] A. Fix, H. Arenhoevel, Eur. Phys. J. A 25, 115 (2005)

[75] Chun Wa Wong, Phys. Rev. C 61, 064011 (2000)

[76] A. Starostin et al., Phys. Rev. C 64, 055205 (2001)

[77] W. Hillert, Eur. Phys. J. A 28, s01 (2006) 139

[78] D.P. Watts et al., Chin. Phys. C33 (2009) 1183-1188

[79] H. Ikeda et. Phys. Rev. Lett. 42, 1321 (1979)

[80] B.A. Mecking et al., Nucl. Inst. and Meth. A 503, 513 (2003)

[81] J. Beringer et al. (Particle Data Group) Phys. Rev. D 86, 010001 (2012) 